\title{
Energy-based Control of a Distributed Solar Collector Field
}

\author{
Tor A. Johansen ${ }^{\text {a }}$ Camilla Storaa ${ }^{\text {a }}$ \\ a Department of Engineering Cybernetics, Norwegian University of Science and \\ Technology, N-74.91 Trondheim, Norway.
}

Model-based control of the outlet temperature of a distributed solar collector field is studied. An energy-based controller is derived using internal energy as a storage function and controlled variable. The controller relies on a distributed parameter nonlinear plant model and includes feedforward from the solar irradiation and inlet temperature. Stability of the closed loop is proved, and the method is experimentally verified to perform well on a pilot-scale solar power plant.

Key words: Model based control; distributed parameter systems; nonlinear control; solar power

\section{Introduction}

The ACUREX-field of Plataforma Solar de Almeria (PSA) is located in the southern part of Spain, see Figure 1 and (Camacho et al. 1997) for a detailed description. The field is composed of 480 distributed solar collectors, arranged in 10 parallel loops. A collector uses the parabolic surface to focus the solar radiation onto a receiver tube, which is placed in the focal line of the parabola, Figure 2. The heat-absorbing fluid (oil) is pumped through the receiver tube, causing the fluid to collect heat which is transferred through the tube surface. The thermal energy developed by the field is pumped to the top of the thermal storage tank, see Figure 1, whereupon the oil from the top of the storage tank can be fed to a power generating system, a desalination plant or to an oilcooling system, if needed. The oil outlet from the storage tank to the field is at the bottom of the storage tank. To ensure that the collectors give high solar absorption, every collector row has a 1 d.o.f. sun tracking system fitted to it.

\footnotetext{
* Corresponding author: Tor.Arne.Johansen@itk.ntnu.no

${ }^{\star}$ Present address of Camilla Storaa: Karolinska Institutet, Department of Medical Engineering, Novum F60, SE-141 86 Huddinge, Sweden.
} 
A control system for this plant has the objective of maintaining the outlet temperature (in this case the average outlet temperature of all the parallel loops) at a desired value in spite of disturbances in solar irradiation (clouds and atmospheric phenomena), irregularities in the sun tracking control system, collector reflectivity and inlet oil temperature. The oil flow rate is manipulated by the control system through commands to the pump. It should be noticed that the primary energy source, solar radiation, cannot be manipulated.

The distributed solar collector field may be described by a distributed parameter model of the temperature (Klein et al. 1974, Rorres et al. 1980, Orbach et al. 1981, Carotenuto et al. 1985, Carotenuto et al. 1985, Camacho et al. 1997). It is widely recognized that the performance og PI and PID type controllers will be inferior to model based approaches (Camacho et al. 1992, Meaburn and Hughes 1995, Camacho et al. 1997). However, the design of a model based controller is not straightforward. The two primary reasons for this is that the plant is highly nonlinear as well as of infinite dimension. Even when the plant is linearized about some operating point and approximated by a finite dimensional model, the frequency response contains anti-resonant modes near the bandwidth that must be taken into consideration in the controller in order to achieve high performance (Meaburn and Hughes 1993, Meaburn and Hughes 1995). Thus, the "ideal" controller should be high-order and nonlinear.

Rorres et al. (1980) and Orbach et al. (1981) suggests an optimal control formulation based on a distributed nonlinear model where the objective is to maximize net produced power when the pumping power is taken into consideration. An alternative approach is taken by (Carotenuto et al. 1985, Carotenuto et al. 1986), where a quadratic control Lyapunov function is formulated for the distributed parameter model, and a stabilizing control law is derived. The approach presented in this paper is similar, but relies on using a storage function with a physical interpretation leading to a conceptually simpler control law with more transparent tuning parameters, see also (Ydstie and Alonso 1997) for a general treatment of thermodynamic storage functions in control.

Other control strategies for this solar power plant based on finite-dimensional models with experimentally evaluated performance can be found in e.g. (Camacho et al. 1997, Silva et al. 1997, Rato et al. 1997, Johansen et al. 2000) and the references therein.

This paper is organized as follows: First we give an overview of the plant and a mathematical model in section 2. Energy-based control strategies are suggested and analysed in section 3 . Some aspects of controller implementation are described in section 4 , and experimental results are included in section 5 before the conclusions. 


\section{Mathematical Model}

The dynamics of the distributed solar collector field are described by the following energy balance

$$
A \frac{\partial T}{\partial t}(t, x)+q(t) \frac{\partial T}{\partial x}(t, x)=\frac{\eta_{0} G}{c \rho} \mu(x) I(t)
$$

with boundary condition $T(t, 0)=T_{\text {in }}(t)$. The position along the collector/tube is $x$ and $t$ is the time. The other model variables are the following

$$
\begin{aligned}
& T(t, x) \text { - oil temperature at position } x \text { along the tube } \\
& q(t) \text { - oil pump volumetric flow rate } \\
& I(t) \text { - solar radition } \\
& \mu(x) \text { - tube/collector characteristic function }
\end{aligned}
$$

Between $x=0$ and $x=l$ the tube contains passive parts that are not exposed to solar radiation, and we have introduced the tube/collector characteristic function $\mu$ to account for this. Hence, $\mu(x)=1$ if the tube at position $x$ is exposed to solar radiation, and $\mu(x)=0$ otherwise. The model parameters are

$$
\begin{aligned}
& A \text { - tube inner cross-sectional area }\left(\mathrm{m}^{2}\right) \\
& \eta_{0} \text { - collector optical efficiency } \\
& G \text { - collector aperture }(\mathrm{m}) \\
& \mathrm{c} \text { - specific oil heat capacity }(\mathrm{J} / \mathrm{K} \cdot \mathrm{kg}) \\
& \rho \text { - oil mass density }\left(\mathrm{kg} / \mathrm{m}^{3}\right) \\
& l \text { - tube length }(\mathrm{m})
\end{aligned}
$$

The model (1) is a somewhat simplified model compared to the models described in (Klein et al. 1974, Rorres et al. 1980, Orbach et al. 1981, Carotenuto et al. 1985, Carotenuto et al. 1986, Camacho et al. 1997). In particular, heat losses and the conductivity of the tube are neglected.

\section{Energy-based control}

The objective is to control the variable $T_{\text {out }}(t)=T(t, l)$ to its specified setpoint. The oil volumetric flow rate $0<q_{\min } \leq q(t) \leq q_{\max }$ is the control input. The upper constraint $q_{\max }$ is due to pump capacity limitations, and the lower constraint $q_{\min }$ is a safety limit in order to reduce the possibility of overheating of the oil. $I(t)$ and $T_{i n}(t)$ can be viewed as measured disturbances. 
Define the internal energy

$$
U(t)=\int_{0}^{l} c T(t, x) \rho A d x
$$

Assuming constant model parameters, the power equation is

$$
\frac{d U}{d t}(t)=\int_{0}^{l} c \frac{\partial T}{\partial t}(t, x) \rho A d x
$$

and from (1)

$$
\begin{aligned}
\frac{d U}{d t}(t) & =\int_{0}^{l}\left(-c \rho q(t) \frac{\partial T}{\partial x}(t, x)+\eta_{0} G \mu(x) I(t)\right) d x \\
& =-c \rho q(t)(T(t, l)-T(t, 0))+\eta_{0} G l_{0} I(t)
\end{aligned}
$$

where

$$
l_{0}=\int_{0}^{l} \mu(\xi) d \xi
$$

is the length of the tube that is exposed to solar radition in the collectors. The interpretation of (5) is that the change in internal energy is balancing the net power transported out of the tube (first term) and the solar power (second term). Assume we define a setpoint profile derived from the reference temperature $T_{\text {out }}^{*}(t)$ :

$$
T^{*}(t, x)=T_{\text {in }}(t)+\left(T_{\text {out }}^{*}(t)-T_{\text {in }}(t)\right) \frac{1}{l_{0}} \int_{0}^{x} \mu(\xi) d \xi
$$

and define the reference internal energy associated with the setpoint profile:

$$
U^{*}(t)=\int_{0}^{l} c T^{*}(t, x) \rho A d x
$$

It is straightforward to show that (7) is a steady-state solution to (1) for some constant $q=q^{*}>0$, provided $I>0, T_{\text {in }}$ and $T_{\text {out }}^{*}>T_{\text {in }}$ are time-invariant. The main idea of the controller is to choose $q(t)$ based on $(5)$ in order to 
explicitly assign a desired linear closed loop dynamic response of $U(t)$, with the consequence that $T_{\text {out }}(t) \rightarrow T_{\text {out }}^{*}$ as $t \rightarrow \infty$ :

Proposition 1 Let $q(t)$ be defined by

$$
\begin{aligned}
& \left.q(t)=\frac{K_{p}}{c \rho(T(t, l)-T(t, 0))}\left(e(t)+T_{d} \frac{d e}{d t}(t)\right)+\frac{1}{T_{i}} \int_{0}^{t} e(\tau) d \tau\right) \\
& e(t)=U(t)-U^{*}(t)=\int_{0}^{l} c\left(T(t, x)-T^{*}(t, x)\right) \rho A d x
\end{aligned}
$$

where $K_{p}, T_{i}>0, T_{d} \geq 0$, and assume $T(t, l)>T(t, 0)$ for all $t$. If $T_{\text {in }}(t), T_{\text {out }}^{*}(t)$ and $I(t) \geq I_{\min }>0$ are time-invariant then i) $U(t) \rightarrow U^{*}$, ii) $T_{\text {out }}(t) \rightarrow T_{\text {out }}^{*}$ and iii) $T(t, x) \rightarrow T^{*}(x)$ for all $x \in[0, l]$ as $t \rightarrow \infty$.

\section{Proof.}

Part i). Combining (9) and (5) we get

$$
\begin{aligned}
\frac{d U}{d t}(t)= & K_{p}\left(U^{*}(t)-U(t)\right)+K_{p} T_{d}\left(\frac{d U^{*}}{d t}(t)-\frac{d U}{d t}(t)\right) \\
& +\frac{K_{p}}{T_{i}} \int_{0}^{t}\left(U^{*}(\tau)-U(\tau)\right) d \tau+\eta_{0} G l_{0} I(t)
\end{aligned}
$$

Laplace transformation of this linear 2 nd order ordinary differential equation leads to

$$
\begin{gathered}
\left(\left(1+K_{p} T_{d}\right) s^{2}+K_{p} s+K_{p} / T_{i}\right) U(s)=\left(K_{p} T_{d} s^{2}+K_{p} s+K_{p} / T_{i}\right) U^{*}(s) \\
+\eta_{0} G l_{0} s I(s)
\end{gathered}
$$

Since $U^{*}(t)$ and $I(t)$ are time-invariant, it follows from (12) that $U(t) \rightarrow$ $U^{*}$ as $t \rightarrow \infty$. Stability of (12) follows from e.g. Hurwitz' criterion since all coefficients of the left-hand-side polynomial are positive.

Part ii). Define the Lyapunov-like functional

$$
V(T)=\frac{1}{2} \int_{0}^{l}\left(T(t, x)-T^{*}(x)\right)^{2} d x
$$

Its time-derivative along trajectories of the system (1) is 


$$
\begin{aligned}
\dot{V} & =\int_{0}^{l}\left(T(t, x)-T^{*}(x)\right) \frac{\partial T}{\partial t} T(t, x) d x \\
& =\int_{0}^{l}\left(T(t, x)-T^{*}(x)\right)\left(-q(t) \frac{\partial T}{\partial x} T(t, x)+\frac{\eta_{0} G}{c \rho} \mu(x) I(t)\right) d x \\
= & -q(t) \int_{0}^{l}\left(T(t, x)-T^{*}(x)\right) \frac{\partial}{\partial x}\left(T(t, x)-T^{*}(x)\right) d x \\
& \quad+\int_{0}^{l}\left(T(t, x)-T^{*}(x)\right)\left(q^{*}-q(t)\right) \frac{\partial T^{*}}{\partial x}(x) d x \\
= & -q(t)\left(T(t, l)-T^{*}(l)\right)^{2}+\Delta(t)
\end{aligned}
$$

where $q^{*}$ and $\Delta(t)$ are defined by

$$
\begin{aligned}
q^{*} \frac{\partial T^{*}}{\partial x}(x) & =\frac{\eta_{0} G}{c \rho} \mu(x) I \\
\Delta(t) & =\left(q^{*}-q(t)\right) \frac{\eta_{0} G}{c \rho} \mu(x) I(t) \int_{0}^{l}\left(T(t, x)-T^{*}(x)\right) d x
\end{aligned}
$$

Due to the exponential convergence of $U(t)$ is it straightforward to see that $\Delta(t) \rightarrow 0$ with exponential convergence as $t \rightarrow \infty$. Note that $q(t)>0$ for $t>t_{0}$ with $t_{0}$ sufficiently large. Integrating (17), the limit of the right hand side of

$$
V(t) \leq V(0)+\int_{0}^{t} \Delta(t) d t
$$

exists and is uniformly bounded and uniformly continuous. It immediately follows that $T(t, l)$ is bounded and $\dot{V}$ is uniformly bounded. We conclude from Barbalat's lemma that $\dot{V} \rightarrow 0$ as $t \rightarrow \infty$. Since $\Delta(t) \rightarrow 0$ as $t \rightarrow \infty$, it follows from (17) that $T(t, l) \rightarrow T^{*}(l)=T_{\text {out }}^{*}$ as $t \rightarrow \infty$.

Part iii). Finally, consider the steady-state solution (18) and define $\delta(t)=$ $q^{*}-q(t)$. Introducing the new variable $\phi(t, x)=T(t, x)-T^{*}(x)$, combining (1) and (18) we get the error equation

$$
\frac{\partial}{\partial t} \phi(t, x)+v \frac{\partial}{\partial x} \phi(t, x)=\varepsilon(t, x)
$$

with boundary condition $\phi(t, 0)=0$, constant flow velocity $v=q^{*} / A>0$, and perturbation 


$$
\varepsilon(t, x)=\frac{1}{A} \delta(t) \frac{\partial}{\partial x} T(t, x)
$$

From the results above, we know $\delta(t) \rightarrow 0$ with exponential convergence as $t \rightarrow \infty$. Since $|U(t)|$ and $\left|\frac{d U}{d t}(t)\right|$ are uniformly bounded and $I, T_{\text {in }}$ and $T_{\text {out }}$ are bounded, it follows that $|\delta(t)|,\left|\frac{\partial}{\partial x} T(t, x)\right|,|T(t, x)|$ and $|\phi(t, x)|$ are uniformly bounded as well, and it is clear that the right hand side of (21) is uniformly bounded and asymptotically vanishing, i.e.

$$
\sup _{x \in[0, l]}|\varepsilon(t, x)| \rightarrow 0 \quad \text { as } t \rightarrow \infty
$$

Note that (21) describes the transport at constant flow velocity $v$. Hence, it is easy to show that the effect of initial conditions is exactly zero for $t>$ $t_{1}=l / v+t_{0}$. Due to $(22)$, for any $\bar{\varepsilon}>0$ there exists a $t_{2} \geq t_{1}$ such that $-\bar{\varepsilon} \leq \varepsilon(t, x) \leq \bar{\varepsilon}$ for all $t \geq t_{2}$ and $x \in[0, l]$. Consider the system

$$
\frac{\partial}{\partial t} \bar{\phi}(t, x)+v \frac{\partial}{\partial x} \bar{\phi}(t, x)=\bar{\varepsilon}, \bar{\phi}(t, 0)=0
$$

defined for $t \geq t_{2}$. It is straightforward to see that with the initial condition $\bar{\phi}(0, x)=f(x)$ where $f(0)=0$ the general solution is $\bar{\phi}(t, x)=\bar{\varepsilon} x / v$ for all $x \in[0, l]$ and $t \geq t_{2}$. A similar result can be dervied for $\phi$ defined by

$$
\frac{\partial}{\partial t} \underline{\phi}(t, x)+v \frac{\partial}{\partial x} \underline{\phi}(t, x)=-\bar{\varepsilon}, \underline{\phi}(t, 0)=0
$$

and we have

$$
-\frac{\bar{\varepsilon} x}{v}=\underline{\phi}(t, x) \leq \phi(t, x) \leq \bar{\phi}(t, x)=\frac{\bar{\varepsilon} x}{v}
$$

Since $\bar{\varepsilon}>0$ can be chosen arbitrarily small, we conclude that $\phi(t, x) \rightarrow 0$ as $t \rightarrow \infty$ for all $x \in[0, l]$.

The feedback (9) is a PID feedback with nonlinear (time-varying) gain. The difference between this PID feedback and PID feedback from the output temperature (Camacho et al. 1992, Meaburn and Hughes 1995, Camacho et al. 1997) must be emphasized. While we consider feedback from internal energy (a concentrated variable containing information about the whole distributed field), the other approaches reported in the literature considers feedback from the outlet temperature (containing only information about a single point in the distributed field). An advantage of our approach is that the dynamics of 
the internal energy are simple (as we have seen it can be assigned low order linear dynamics) compared to the dynamics of the outlet temperature (infinite order and non-linear with anti-resonant modes).

The assumptions $T(t, l)>T(t, 0)$ and $I(t) \geq I_{\min }>0$ are non-restrictive since they will always hold during normal operation of the plant. The reason for this is that the purpose of the plant is to produce energy in terms of increased temperature of the oil. The above assumption will not necessarily hold at startup and when that solar radition is very low for a long time, but to handle such cases it is common practice shut down the plant when the solar power is very low for a long time, and in other abnormal situations to rely on a supervisory system that overrides the controller that is used during normal operation.

Adding a feedforward to this control strategy will be beneficial from a disturbance rejection performance point of view, and is common in this plant since $T_{i n}(t)$ and $I(t)$ are both measured, (Camacho et al. 1997). One may design from (5) a feedforward control $q_{f f}(t)$ that cancels (at steady-state) the supplied solar power as follows

$$
q_{f f}(t)=\frac{\eta_{0} G l_{0}}{c \rho\left(T_{\text {out }}(t)-T_{\text {in }}(t)\right)} I(t)
$$

The main theoretical properties of the control system remain unchanged, also when replacing $T_{\text {out }}(t)$ with $T_{\text {out }}^{*}(t)$ in $(27)$ :

Proposition 2 Let $q(t)$ be defined by either

$$
\begin{aligned}
q(t)= & \frac{\eta_{0} G l_{0}}{c \rho\left(T_{\text {out }}(t)-T_{\text {in }}(t)\right)} I(t) \\
& \left.\quad+\frac{K_{p}}{c \rho(T(t, l)-T(t, 0))}\left(e(t)+T_{d} \frac{d e}{d t}(t)\right)+\frac{1}{T_{i}} \int_{0}^{t} e(\tau) d \tau\right)
\end{aligned}
$$

or

$$
\begin{aligned}
q(t)= & \frac{\eta_{0} G l_{0}}{c \rho\left(T_{\text {out }}^{*}(t)-T_{\text {in }}(t)\right)} I(t) \\
& \left.\quad+\frac{K_{p}}{c \rho(T(t, l)-T(t, 0))}\left(e(t)+T_{d} \frac{d e}{d t}(t)\right)+\frac{1}{T_{i}} \int_{0}^{t} e(\tau) d \tau\right)
\end{aligned}
$$

where $K_{p}, T_{i}>0, T_{d} \geq 0$, and assume $T(t, l)>T(t, 0)$ for all $t$. If $T_{\text {in }}(t), T_{\text {out }}^{*}(t)$ and $I(t) \geq I_{\min }>0$ are time-invariant, then i) $U(t) \rightarrow U^{*}$, ii) $T_{\text {out }}(t) \rightarrow T_{\text {out }}^{*}$ and iii) $T(t, x) \rightarrow T^{*}(x)$ for all $x \in[0, l]$ as $t \rightarrow \infty$. 
Proof. Consider first (29). The additional feedforward term is time-invariant under the stated assumptions and the power equation reduces to (cf. (11)):

$$
\frac{d U}{d t}(t)=K_{p}\left(U^{*}(t)-U(t)\right)+K_{p} T_{d}\left(\frac{d U^{*}}{d t}(t)-\frac{d U}{d t}(t)\right)+\frac{K_{p}}{T_{i}} \int_{0}^{t}\left(U^{*}(\tau)-U(\tau)\right) d \tau
$$

since the feedforward cancels the solar power. The rest of the proof is similar to the proof of Proposition 1.

Next, consider (28). In this case it can be seen that the power equation can be written

$$
\frac{d U}{d t}(t)=-c \rho q_{f b}(t)(T(t, l)-T(t, 0))+\eta_{0} G l_{0} I-c \rho q^{*}(T(t, l)-T(t, 0))
$$

where

$$
q_{f b}(t)=\frac{K_{p}}{c \rho(T(t, l)-T(t, 0))}\left(e(t)+T_{d} \frac{d e}{d t}(t)+\frac{1}{T_{i}} \int_{0}^{t} e(\tau) d \tau\right)
$$

which conincides with (9). It is clear that (31) is equivalent to the power equation (11) derived in the proof of Proposition 1 since the last term in (31) can be taken into the inital value of the integrator in the PID controller. Hence, the result follows from Proposition 1.

\section{Controller implementation}

The implementation of the controller (29) contain in addition to the feedback and feedforward described above the following components

- A reference filter.

- An open-loop observer to estimate the temperature distribution.

- An outer feedback loop with integral action in order to reduce steady-state error, i.e. compensate for unmodelled dynamics and unmeasured disturbances.

- To avoid singularity of the controller, we set $q(t)=q_{\min }$ when $T_{\text {out }}^{*}(t) \leq$ $T_{\text {in }}(t)$ or $T_{\text {out }}(t) \leq T_{\text {in }}(t)$. 
The observer contains a real-time numerical integration of the distributed plant model (1), with the modification that heat losses due to conduction are accounted for. This gives $\hat{T}(t, x)$, the estimated temperature in the tube. Spatial discretization intervals at $1 \mathrm{~m}$ and temporal discretization intervals at $0.5 \mathrm{~s}$ is utilized in the finite difference numerical integration. Nominal stability of the closed loop with the observer in the case of constant disturbances can be established since $\hat{T}(t, x)=T(t, x)$ regardless of initial conditions for $t$ sufficiently large (recall that the plant is open loop stable and the observer is an open loop observer).

The outer feedback loop has the structure

$$
T_{\text {out }}^{*}(t)=K_{p}^{\circ}\left(\Delta T(t)+\frac{1}{T_{i}^{o}} \int_{0}^{t} \Delta T(\tau) d \tau\right)
$$

where $\Delta T(t)=\hat{T}_{\text {out }}(t)-T_{\text {out }}(t)$ and $\hat{T}_{\text {out }}(t)$ is the filtered commanded outlet temperature. The outer feedback loop PI-parameters are $K_{p}^{o}=1.25$ and $T_{i}^{o}=$ $400 \mathrm{~s}$. Due to the saturation limits of the pump at $q_{\min }=2 \mathrm{l} / \mathrm{s}$ and $q_{\max }=$ $10 \mathrm{l} / \mathrm{s}$, a simple anti-windup strategy is implemented for (33) and (29). This essentially turns off the integrators while the control input is saturated.

The nonlinear PID parameters are chosen as $K_{p}=1.8 \mathrm{l} / \mathrm{s} / \mathrm{m}^{3}, T_{d}=66 \mathrm{~s}$ and $T_{i}=210 \mathrm{~s}$. The sampling interval of the control system is $15 \mathrm{~s}$.

\section{$5 \quad$ Experimental results}

Figures $3-5$ show the results of three experiments. In all cases 9 out of 10 loops of the collector field are active. The controlled variable $T_{\text {out }}(t)$ is the average outlet temperature of these 9 loops.

The scenario in Figure 3 consists of several step changes in the reference temperature. We observe that the response is fast with no significant overshoot or steady-state error, except for the startup phase where we note that there are significant disturbances in the inlet temperature $T_{i n}(t)$ as well as the solar irradition $I(t)$ that increases rapidly.

In Figure 4 the reference temperature is constant for most of the period. At around $14: 20$ a small cloud leads to a short interval with almost zero irradiation. The controller responds by reducing the flow rate to $q_{\text {min }}$ in order to minimize the impact of the disturbance on the internal energy. We note that the controller quickly re-establishes the equilibrium after the disturbance. 
The results in Figure 5 shows the response to a large increase in the inlet temperature. A $30 \mathrm{~K}$ peak inlet disturbance is damped to a peak of about about $7 \mathrm{~K}$ at the outlet. It was realised after the experiments that the inlet piping dimensions were not accurate in the model, so we expect that improved disturbance rejection performance could be achieved by refining the model.

Compared to experimental results from the literature for this plant, see (Camacho et al. 1997) for results with a wide range of controllers, we conclude that the performance achieved with the energy-based controller is similar to the best model-based controllers, and better than fine-tuned fixed PI and PID controllers (compare with the results in section 3.4 of (Camacho et al. 1997) and (Meaburn and Hughes 1995) page 139).

\section{Conclusions}

We have presented a nonlinear controller for a solar power plant based on an distributed parameter model of the collector field. A conceptually simple control design based on controlling the internal energy of the plant is suggested. In addition to achieving high performance and robustness, the main advantage of this approach is that it allows simple and transparent tuning of the nonlinear controller through some PID parameters, and a stability proof is provided.

\section{Acknowledgements}

We gratefully acknowledge the support of the European Commission through

"Access to Research Infrastructure action of the Improving Human Potential Programme". We are also grateful for the excellent support provided by Loreto Valenzuela and the rest of the staff at Plataforma Solar de Almeria.

\section{References}

Camacho, E. F., M. Berenguel and F. R. Rubio (1997). Advanced Control of Solar Plants. Springer-Verlag, London.

Camacho, E. F., R. F. Rubio and F. M. Hughes (1992). Self-tuning PI control of a solar power plant with a distributed collector field. IEEE Control Systems Magazine 12(2), 72-78. 
Carotenuto, L., M. La Cava and G. Raiconi (1985). Regulator design for the bilinear distributed parameter of a solar power plant. Int. J. Systems Science 16, 885900 .

Carotenuto, L., M. La Cava, P. Muraca and G. Raiconi (1986). Feedforward control for the distributed parameter model of a solar power plant. Large Scale Systems 11, 233-241.

Johansen, T. A., K. J. Hunt and I. Petersen (2000). Gain scheduled control of a solar power plant. Control Engineering Practice 8, 1011-1022.

Klein, S. A., J. A. Duffie and W. A. Beckman (1974). Transient considerations of flat-plate solar collectors. Trans. ASME J. Engng. Power 96A, 109-.

Meaburn, A. and F. M. Hughes (1993). Resonance characteristics of distributed solar collector fields. Solar Energy 51, 215-221.

Meaburn, A. and F. M. Hughes (1995). Pre-scheduled PID control of a solar thermal power plant. Transactions of the Institute of Measurement and Control 17, 132142.

Orbach, A., C. Rorres and R. Fischl (1981). Optimal control of a solar collector loop using a distributed-lumped model. Automatica 17, 535-539.

Rato, L., D. Borrelli, E. Mosca, J. M. Lemos and P. Balsa (1997). MUSMAR based switching control of a solar collector field. In: Proceedings of the European Control Conference, Brussels.

Rorres, C., A. Orbach and R. Fischl (1980). Optimal and suboptimal control policies for a solar collector system. IEEE Trans. Automatic Control 25, 1085-1091.

Silva, R. N., L. M. Rato, J. M. Lemos and F. Coito (1997). Cascade control of a distributed collector solar field. J. Process Control 7, 111-117.

Ydstie, B. E. and A. A. Alonso (1997). Process systems and passivity via the Clausius-Planck inequality. Systems and Control Letters 30, 253-264. 


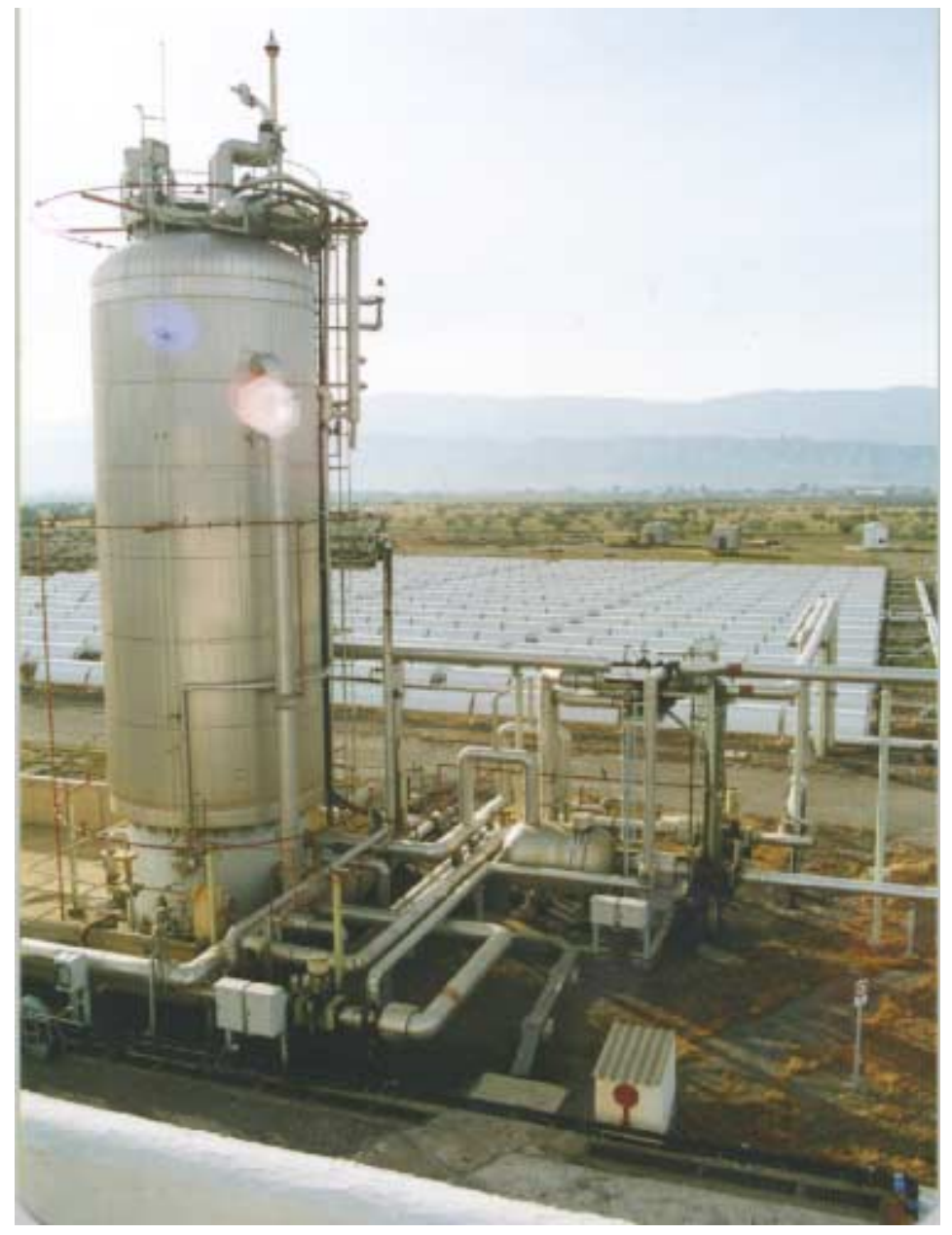

Fig. 1. ACUREX, the distributed collector field at PSA, Almeria, Spain.

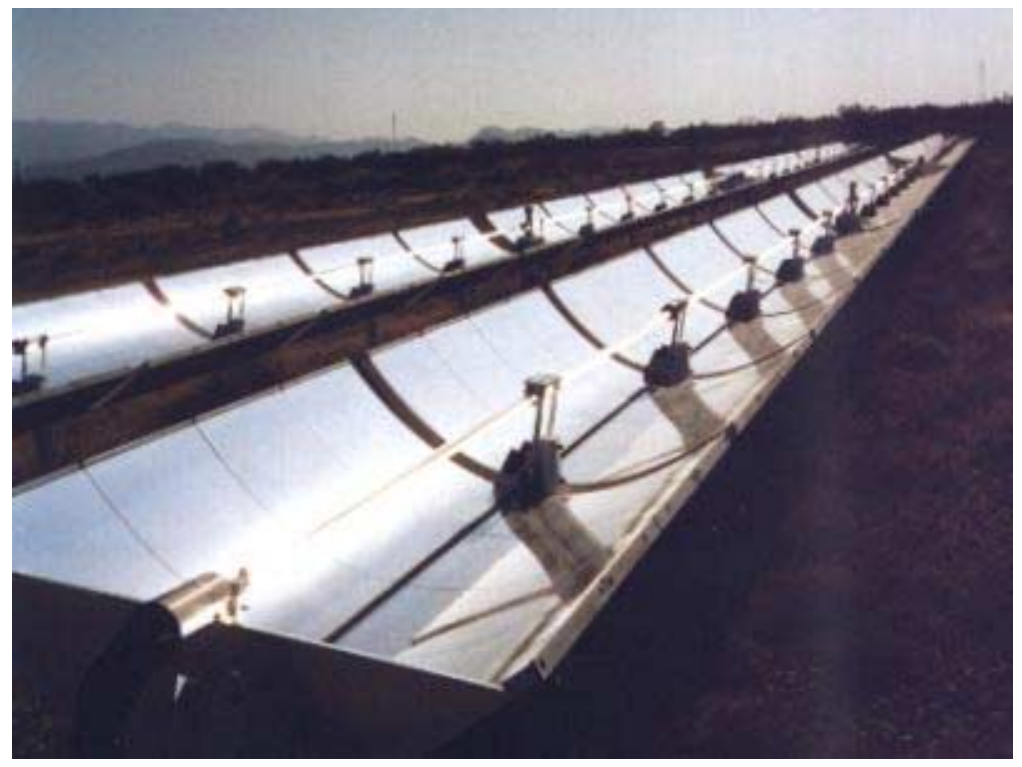

Fig. 2. Parabolic collector. 

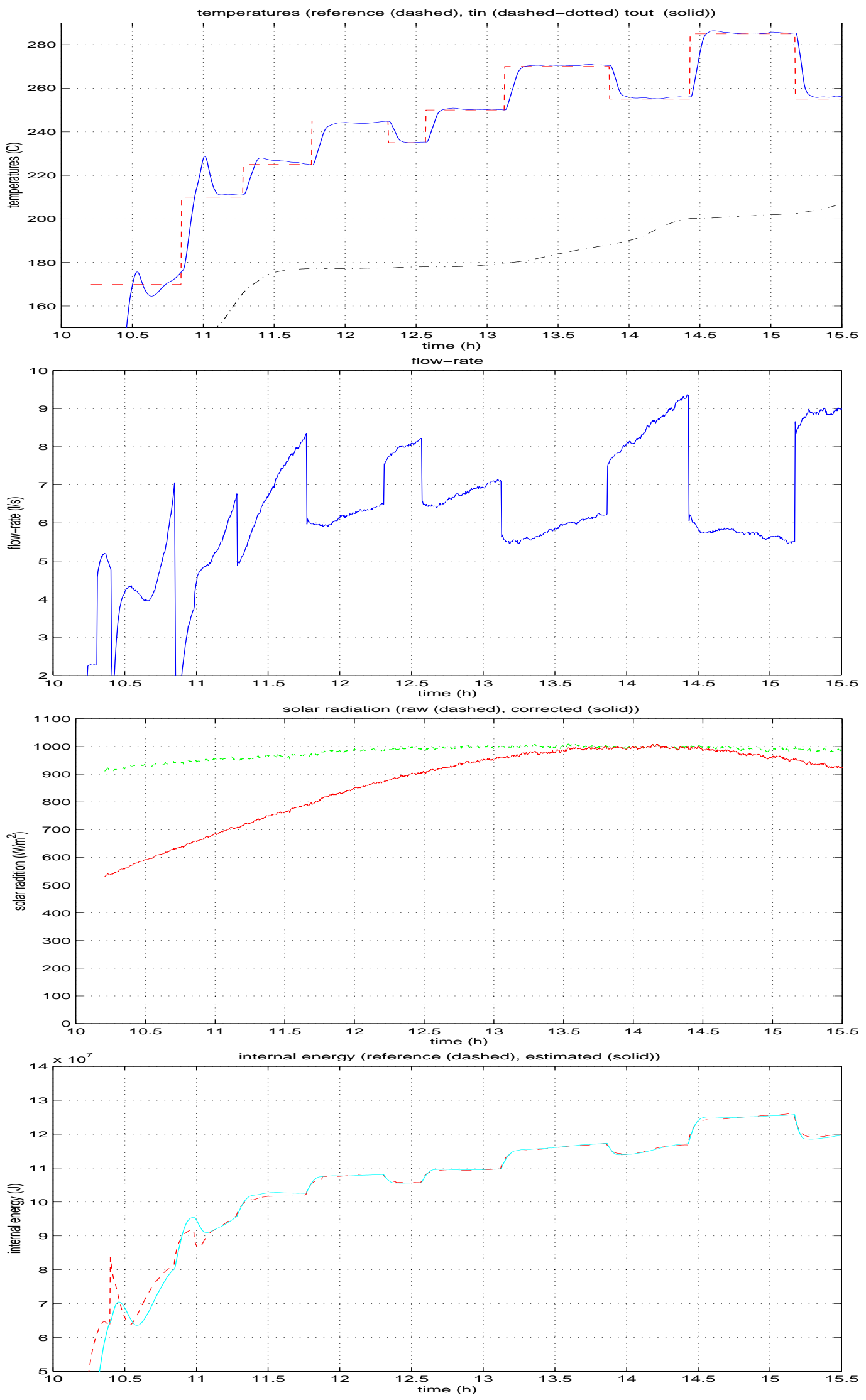

Fig. 3. Experimental results 28 May 2001. 

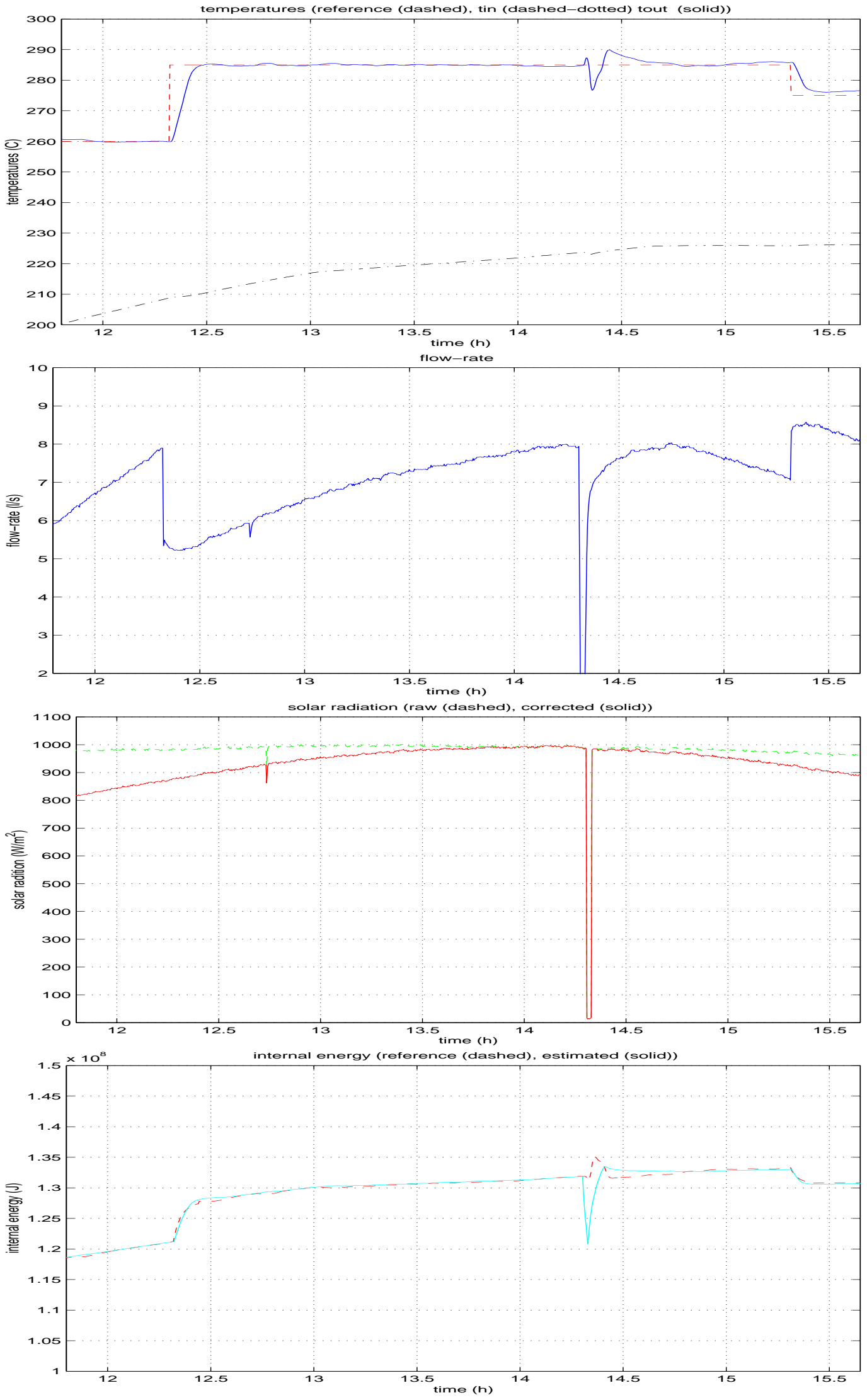

Fig. 4. Experimental results 30 May 2001. 

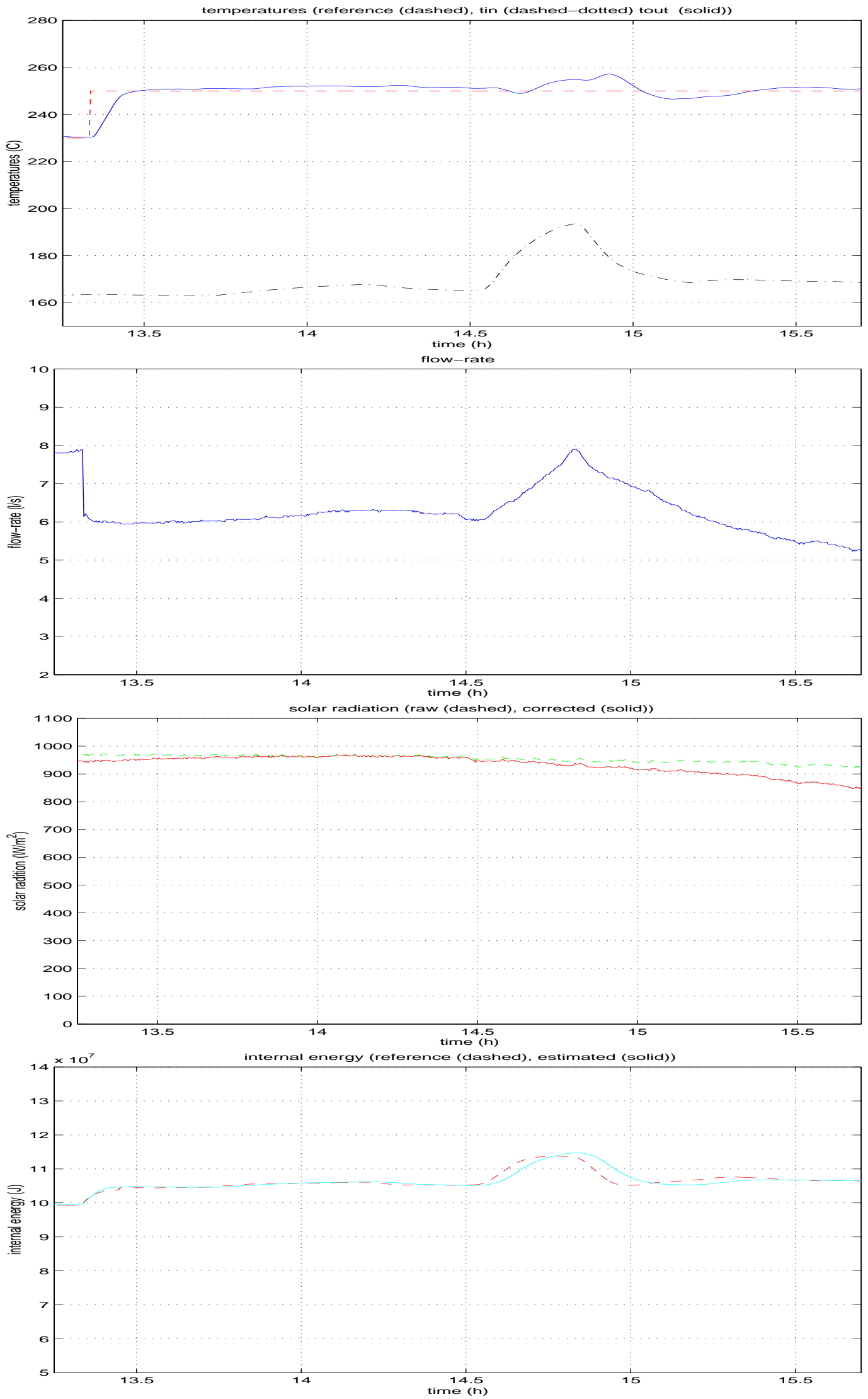

Fig. 5. Experimental results 31 May 2001. 